\title{
Importance of Meta-Analysis and Practical Obstacles in Oncological and Epidemiological Studies: Statistics Very Close but Also Far!
}

\author{
Ozgur Tanriverdi ${ }^{1}$, Nese Yeniceri ${ }^{2}$
}

\begin{abstract}
Studies of epidemiological and prognostic factors are very important for oncology practice. There is a rapidly increasing amount of research and resultant knowledge in the scientific literature. This means that health professionals have major challenges in accessing relevant information and they increasingly require best available evidence to make their clinical decisions. Meta-analyses of prognostic and other epidemiological factors are very practical statistical approaches to define clinically important parameters. However, they also feature many obstacles in terms of data collection, standardization of results from multiple centers, bias, and commentary for intepretation. In this paper, the obstacles of meta-analysis are briefly reviewed, and potential problems with this statistical method are discussed.
\end{abstract}

Keywords: Meta-analysis - obstacles - oncology - cancer research

Asian Pac J Cancer Prev, 16 (3), 1303-1306

\section{Introduction}

There are increasing number of researches and increasing amount of knowledge in scientific literature and health care providers have challenges to reach accurate information and they increasingly require to make their decisions on the best available evidence. Evidence-based medicine (EBM) with applying "current best evidence to decisions on the care of individual patients" and knowledge synthesis have become increasingly important for clinical decision-making (Kranke, 2010). The "best evidence" can be gathered by reading randomized controlled trials (RCTs), systematic reviews, and meta-analyses providing an unbiased overview of increasing amount of knowledge on a particular subject (Noordzij et al., 2009; Impellizzeri et al., 2012; Khan et al., 2014a). Meta-analysis are often considered as the highest level of evidence in health care (Lyman and Kuderer, 2005).

Sackett et al defined EBM as "the integration of the best research evidence with clinical expertise and the patient's preferences and values" (Sackett et al., 2000). This definition highlights the relationship between research evidence and other factors including patient values and preferences and clinical circumstances which are integral to evidence-based decision making and that research evidence alone is not sufficient for Evidincebased decision making. Clinical circumstances include all the information of the patient's health concerns, complicating clinical conditions and the disease course estimated survival, whereas patient preferences and values include their health beliefs, risk tolerance, ethical and religious beliefs that can effect treatment options and health choices (Tilburt, 2008; Khan et al., 2014b). Clinical circumstances and patient values should be taken into account for evidence-based decision making (Saxena et al., 2012).

Systematic reviews synthesise the world literature systematically, also known as the "research synthesis" providing a summary of medical reports on a particular subject using explicit methods to search (Sackett et al., 2000; Tricco et al., 2011). It also aims to provide a comprehensive, unbiased synthesis of many relevant studies in a single document (Tricco et al., 2011). Systematic reviews bring together a number of individual studies that viewed separately, sometimes with conflicting findings and inconclusive results due to a relatively small sample size and synthesize their results (Akaberg, 2005; Noordzij et al., 2009). Meta-analysis is a mathematical synthesis of the results of these individual studies and obtains more reliable information about treatment effects (Noordzij et al., 2009). In meta-analysis, data from individual studies are reanalysed using established statistical methods in other words it synthesizes the results of the individual studies into a new result (Noordzij et al., 2009; Gonzalez et al., 2011; Impellizzeri et al., 2012). It is not a simple arithmetic average of the results of different studies, but a weighted average (Akaberg, 2005). By combining those individual studies in a meta-analysis, the overall sample size is increased so the statistical power of the analysis is increased, resulting in the precision of 
the estimates of treatment effects (Akaberg, 2005). Metaanalysis can increase power and precision of estimates of treatment effects (Akaberg, 2005). It investigates the reported results of the studies in all aspects; theoretical constructs, operational definitions of the independent or manuplated variable, moderating and mediating variables, and dependent variables, population samples, data collection procedures, statistical analyses and especially the handling of possible confounding variables that would provide an alternative explanation for the reported results (Hoble, 2008). Most of the meta-analyses focus on the effects of therapy or prevention and they usually based on RCTs because they are more likely to provide unbiased information than other study designs (Noordzij et al., 2012).

Meta-analyses are very common in cancer studies and in oncology, It have a long tradition and continue to play an important role as they produce important contributions to clinical practice (Berman and Parker, 2002; Columb and Lakhen, 2005; Zwahlen et al., 2008). To give an example, The Early Breast Cancer Trialists Collaborative Group showed that tamoxifen improves the 10-year survival of women with reductions in breast cancer recurrence and mortality (EBCTCG, 1998).

\section{Practical Obstacles in Meta-analysis}

There has been some debate on whether meta-analyses provide reliable evidence. Meta-analyses with limited evidence, biased studies, and poor-quality trials are considered to have unreliable results so assessing the quality of clinical evidence appears to be very important (Ioannidis, 2005). In a study which assessed the quality of the sources(reviews, systematic reviews and metaanalyses) used for the development of guidelines on prevention and treatment of breast and colorectal cancers, it is found that only $30.3 \%$ of breast cancer and $26.1 \%$ of colorectal cancer guidelines used at least one high quality review (Vigna-Taglianti et al., 2006). In another study, The Appraisal of Guidelines and Research and Evaluation (AGREE) Instrument was used to assess the quality of 100 guidelines (including 32 oncology guidelines) from 13 countries and showed that oncology guidelines had significantly higher scores on rigor of development than nononcology guidelines (42.2\% versus $29.4 \%$; $\mathrm{P}=0.02$ ) (Burgers et al., 2004). To improve the quality of Metaanalyses, there have been efforts to establish guidelines and standards for reporting and it's tecniques (Noordzij et al., 2009; Engberg, 2008; Pace and Stat, 2011) and it's likely to increase the proportion of true findings (Ioannidis, 2005). Quality of Reporting of Meta-Analyses (QUORUM) statement which was published in 1999, describes the preferred way to present the Abstract, Introduction, Methods, Results and Discussion sections of a report of a meta-analysis and provides information about the number of RCTs identified, included and excluded, and the reasons for exlusion (Noordzij et al., 2009).

The major issues regarding meta-analysis can be classified as questions of heterogeneity and questions of bias (Ioannidis, 2008). Meta-analyses are very valuable especially when the results of the studies they include show clinically important effects of similar magnitude, onthe other hand, when the included studies have differing results, the conclusions are less clear (Higgins et al., 2003). Statistical heterogeneity exists when the true effects being evaluated differ between studies. There is clinical and methodological heterogeneity in meta-analyses as there are clinical diversities of study participants like age, sex, concominant diseases and the delivery of the interventions like drug dose and associated therapies as well as variability in trial design and quality (Pace and Stat, 2011). If studies are clinically too diverse, the results of a meta-analysis may be meaningless, so assessment of the consistency of effects among studies is very important (Higgins et al., 2003; Noordzij et al., 2009). The findings of the meta-analysis cannot be generalised unless we know how consistent the results of studies are. On the other hand, several hierarchical systems for grading evidence state that the results of studies must be consistent or homogeneous to obtain the highest grading (Higgins et al., 2003).

Statistical tests are available and should be performed to quantify heterogeneity (Gonzalez et al., 2011; Columb and Lakhen, 2005). A quantity called " $\mathrm{I}$ " " was developed which quantifies the effect of heterogeneity, providing a measure of the degree of inconsistency in the studies' results (Higgins et al., 2003). It also describes the percentage of total variation across studies that is due to heterogeneity rather than chance (Higgins et al., 2003). It takes values from 0 to $100 \%$ and a value of $0 \%$ indicates no observed heterogeneity, and larger values Show increasing heterogeneity (Higgins et al., 2003). Often cut-offs are used to claim the degree of heterogeneity, for example, $50 \%$ is the cut-off for large heterogeneity (Ioannidis, 2008).

Possible source of heterogeneity in meta-analyses can be explored using sensitivity, subgroup or regression analyses (Impellizzeri et al., 2012). If heterogeneity is identified in a meta-analysis a common option is to subgroup the studies. Subgroup analysis, seeks factors in some studies that systematically modify the treatment effect (Pace and Stat, 2011).

Sensitivity analysis is a component of a meta-analysis which provides to test the sensitivity of the summary estimate of the treatment in other words to test the strength of the main findings (Columb and Lakhen, 2005; Pace and Stat, 2011).

There are two points of view in the statistical calculation of the summary effect and combining the results; the 'fixed-effect' model and the 'random-effects' model (Impellizzeri et al., 2012; Pace and Stat, 2011). In the fixed-effect model, it is assumed that the variability between studies is only due to chance variation, in other words, the group of studies give an estimate of the same treatment effect (Impellizzeri et al., 2012; Pace and Stat, 2011). In the random-effects model, it is assumed that there is heterogeneity among studies and asumes that no single treatment effect exists, but that each study has a different true effect (the true effect varies from study to study) (Noordzij et al., 2009; Pace and Stat, 2011; Impellizzeri et al., 2012). Both fixed and random-effects models are used for examining the robustness of the analysis (Impellizzeri et al., 2012). When severe heterogeneity is detected, the 
meta-analysis should be aborted (Impellizzeri et al., 2012). Heterogeneity tests are routinely applied in almost all meta-analyses and tests for publication bias are also used extensively (Ioannidis, 2008).

A major problem for meta-analyses is a potential bias in the data. There are many terms used to describe biases related to the selective dissemination of evidence, such as language bias, funding bias, database bias, citation bias, location bias, reviewer bias (Bax and Moons, 2011) and outcome reporting bias (selective reporting of positive outcomes). These forms of biases tend to have more effect on small studies and contribute to the phenomenon of "small study-effects" (van Enst et al., 2014). This means that published studies with small sample sizes tend to have larger and more favourable effects compared to studies with larger sample sizes. This is a threat to the validity of a systematic review and its meta-analyses (Thornton and Lee, 2000).

Publication bias is traditionally considered the major threat (Ioannidis, 2008). Studies with 'negative' (nonstatistically significant) results are more likely to be underpresented and remain unpublished than studies with 'positive' (formally statistically significant) results, therefore the results of a literature research can be expected to be biased toward significant results and eventually anticipated results (Ioannidis, 2008; Savelova and Selinski, 2008). Standard methods to detect a publication bias in a meta-analysis are a visual inspection of the funnel plot and the application of the Egger's regression test (Savelova and Selinski, 2008). In the funnel plot, study sizes or standard errors are plotted against estimates of the effect size (for example Odds Ratios) (Savelova and Selinski, 2008). The meta results are shown in the funnel plot as a vertical line (Savelova and Selinski, 2008). In the absence of bias, the plot sould resemble a 'funnel shape', in the presence of publication bias, some smaller studies reporting negative results will be missing and an asymmetrical funnel plot will be observed thus publication bias is not the only possible reason for observed funnel plot asymmetry (Peters et al., 2006). Egger's and Begg's regression tests are commonly used statistical tests for publication bias (van Enst et al., 2014).

The Cochrane Handbook for Systematic Reviews of Diagnostic Test Accuracy explicitly mentions not to use methods like the Begg or Egger tests and argues that it is best to use the test proposed by Deeks. A recent simulation study in DTA meta-analyses showed that the trim and fill method is more powerful than other tests like the Begg, Egger or Deeks test to detect possible publication bias. Therefore, this method may be used more frequently in future (van Enst et al., 2014).

\section{Conclusions and Recommendations}

When conducting a meta-analysis one should also consider recently developed methods to detect a potential bias. Investigators should be aware of potential biases such as poor quality of included studies, heteregeneity between studies, and presence of publication and outcome reporting bias.

\section{References}

Akobeng AK (2005). Understanding systematic reviews and meta-analysis. Arch Dis Child, 90, 845-8.

Bax L, Moons KG (2011). Beyon publication bias. J Clin Epidemiol, 64, 459-462

Berman NG, Parker RA (2002). Meta-analysis: Neither quick nor easy. BMC Med Res Methodol, 2, 10.

Burgersm JS, Fervers B, Haugh M, et al (2004). International assessment of the quality of clinical practice guidelines in oncology using the appraisal of guidelines and research and evaluation instrument. J Clin Oncol, 22, 2000-7.

Columb MO, Lalkhen AG (2005). Systematic reviews and meta-analyses. Current Anesthesia Critical Care, 16, 391-4.

Early Breast Cancer Trialists' Collaborative Group (1998). Tamoxifen for early breast cancer: an overview of the randomised trials. Lancet, 35, 1451-67.

Engberg S (2008). Systematic reviews and meta-analysis. $J$ Wound Ostomy Continence Nurs, 35, 258-265.

Gonzalez IF, Urrutia G, Alonso-Coello P (2011). Systematic Reviews and Meta-Analysis: Scientific rationale and interpretation. Rev Esp Cardiol, 64, 688-96.

Higgins JP, Thompson SG, Deeks JJ, Altman D (2003). Measuring inconsistency in meta-analyses. BMJ, 327, 557-560.

Impellizzeri FM, Bizzini M (2012). Systematic review and meta-analysis: a primer. Int J Sports Phys Ther, 7, 493-503.

Ioannidis JP (2005). Why most published research findings are false. PLos Med, $2,124$.

Ioannidis JP (2008). Interpretation of tests of heterogeneity and bias in meta-analysis. J Eval Clin Pract, 14, 951-7.

Khan HFR, Saxena A, Gabbidon K, Ross E, Shrestha A(2014a). Statistical applications for the prediction of white Hispanic breast cancer survival. Asian Pac J Cancer Prev, 15, 5571-5.

Khan HFR, Saxena A, Rana S, Ahmed NU (2014b). Bayesian method for modeling male breast cancer survival data. Asian Pac J Cancer Prev, 15, 663-9.

Kranke P (2010). Evidence-based practice: how to perform and use systematic reviews for clinical decision-making. Eur $J$ Anaesthesiol, 27, 763-72.

Lyman GH, Kuderer NM (2005). The strengths and limitations ofmeta-analyses based on aggregate data. BMC Med Res Methodol, 5, 14.

Noble JH (2006). Meta-analysis: Methods, strengths, weaknesses and political uses. J Lab Clin Med, 147, 7-20.

Noordzij M, Hooft L, Dekker FW, Zoccali J, Jager K.J (2009). Systematic reviews and meta-analyses: when they are useful and when to be careful. Kidney Int, 76, 1130-6.

Pace NL, Stat M (2011). Research methods for meta-analyses. Best Practice and Res Clinical Anesthesiol, 25, 523-33.

Peters JL, Sutton AJ, Jones DK, Abrams KR, Rushton R (2006) Comparison of two methods to detect publication bias in meta-analysis, JAMA, 295, 670-680.

Sackett DL, Srs SE, Richardson WS, Rosenberg W, Haynes RB. Evidence-Based Medicine: How to Practice and Teach EBM, 2nd edn. 2000, Edinburgh: Churchill Livingstone.

Saveleva E, Selinski S (2008). Meta-analyses with Binary Outcomes: how many studies need to be omitted to detect a publication bias? J Toxicol Environ Healt, 71, 845-50.

Saxena U, Sauvagelt C, Sankaranarayanan R (2012). Evidencebased screening, early diagnosis and treatment strategy of cervical cancer for National Policy in low-resource countries:example of India. Asian Pac J Cancer Prev, 13, 1699-1703.

Thornton A, Lee P (2000). Publication bias in meta-analysis: its causes and consequences. J Clin Epidemiol, 53, 207-216.

Tilburt JC (2008). Evidence-based medicine beyond the 
Ozgur Tanriverdi and Nese Yeniceri

bedside:keeping an eye on context. J Eval Clin Pract, 14, $721-5$.

Tricco AC, Tetzlaff J, Moher D(2011). The art and science of knowledge synthesis. J Clin Epidemiol, 64, 11-20.

van Enst MA, Ochodo E, Scholten R, Hooft L (2014). Investigation of publication bias in meta-analyses of diagnostic test accuracy: a meta-epidemiological study. $B M C$ Med Res Methodol, 14, 70.

Vigna-Taglianti F, Vineis P, Liberati A, Faggiano f(2006). Quality of Systematic Reviews used in guidelines for oncology practice. Ann Oncol, 17, 691-701.

Wu YZ, Yang H, Zhang L, et al (2012). Application of crossover analysis-logistic regression in the assessment of geneenviromental interactions for colorectal cancer. Asian Pac J Cancer, 13, 2031-7.

Zwahlen M, Renehan A, Egger M (2008). Meta-analysis in medical research: Potentials and limitations. Urol Oncol, 26, 320-9. 